\title{
Staging of breast cancer with PET/CT
}

\author{
J S Möller, FCNP (SA) \\ W H Pieterse, $M$ Med (Rad D) \\ P D du Toit, $M$ Med Sc \\ Free State PET/CT Centre, Bloemfontein \\ $S$ Bonnet, $M$ Med (Rad T) \\ A Diedericks, FCOG (SA) \\ W Brummer, M Med (Anat Path) \\ Bloemfontein Medi-Clinic
}

Imaging plays a vital role in the staging and restaging of breast cancer. Traditionally, chest X-ray, bone scintigraphy and liver ultrasound or computed tomography are used. ${ }^{1}$ Fluorine-18 fluorodeoxyglucose (F-18 FDG) positron emission tomography/computed tomography (PET/CT) has great potential for whole-body staging with a single procedure. The greatest utility of PET/CT lies in distant or M-staging, whereas its role in locoregional staging is generally considered to be complementary to other modalities. ${ }^{2,3}$

We present details of a 48 -year-old woman with breast carcinoma, clinically staged as a T3N2 lesion. Initial ultrasonography showed a liver lesion with an equivocal appearance. A F-18 FDG PET/CT scan was then performed. It showed a left breast mass with two pathological axillary lymph nodes. The breast tumour and lymph nodes were associated with intense FDG-activity. A hypodense liver lesion with absent FDG activity was noted, consistent with a haemangioma (not shown). With the pelvic images, a myomatous uterus was seen with a small mass right posterolateral to the uterus. The mass measured $19 \mathrm{~mm}$ x $14 \mathrm{~mm}$, with a density

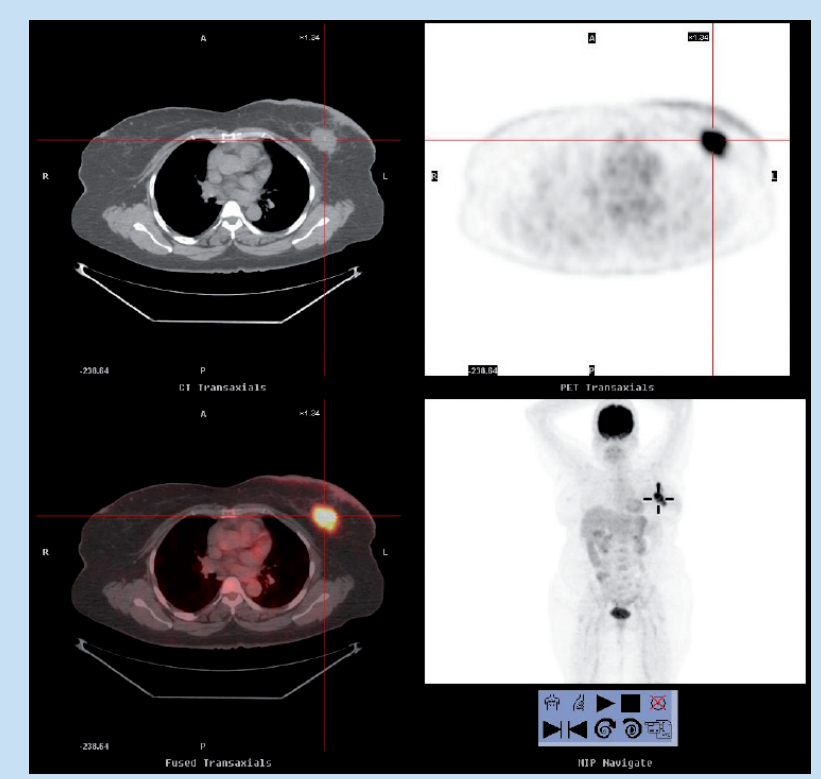

Fig. 1. Transaxial PET/CT images of the primary breast tumour. of 80 Hounsfield units and increased metabolic activity. After review, the differential diagnosis was finally proposed as follows: primary ovarian lesion, pedunculated uterine myoma with sarcomatous degeneration and an ovarian metastasis.

A hysterectomy with bilateral salpingo-oophorectomy was performed. Metastatic breast carcinoma was seen in the ovary with

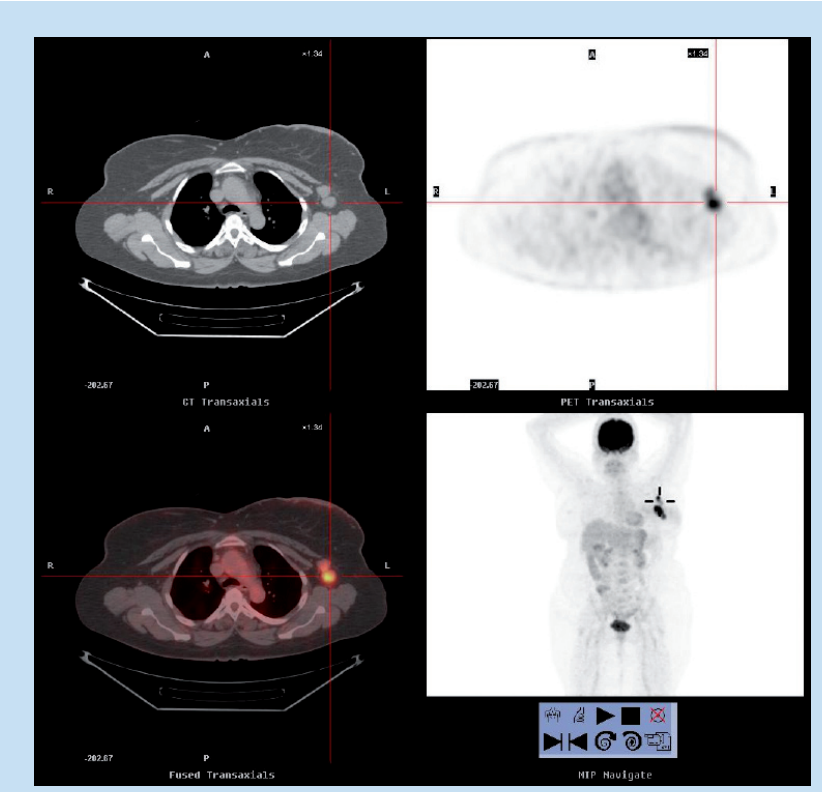

Fig. 2. Axillary lymphadenopathy, with abnormal metabolic activity.

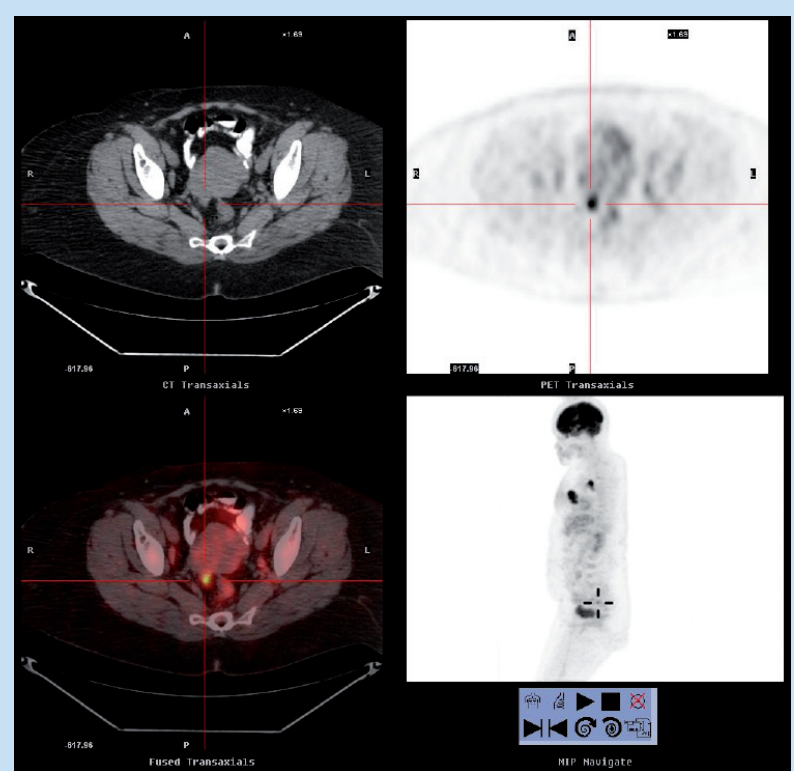

Fig. 3. Small, metabolically active mass postero-lateral to the uterus. 


\section{PICTORIAL ESSAY}

micrometastases in the contralateral ovary. It is known that PET may miss micrometastases. ${ }^{4}$ The patient was upstaged to M1 with a subsequent change in management. Following chemotherapy, a toilet mastectomy and axillary clearance were performed. Histology confirmed an infiltrating ductal carcinoma with metastatic carcinoma in 5 of the 10 axillary lymph nodes. Post-surgical radiotherapy was not indicated in view of the M1 status. The patient is currently on hormonal therapy and in remission 12 months later.

These findings illustrate the utility of F-18 FDG PET/CT in the staging of breast carcinoma, by clarifying an equivocal liver lesion and demonstrating an occult ovarian metastasis. However, this report also shows that the sensitivity of PET/CT regarding micrometastases is limited.

1. Buscombe JR, Holloway B, Roche N, et al. Position of nuclear medicine imaging modalities in the diagnostic work-up of breast cancer. Q J Nucl Med Mol Imaging 2004; 48: 109-118.

2. Eubank WB, Mankoff DA. Current and future uses of positron emission tomography in breast cancer imaging. Semin Nucl Med 2004; 34: 224-240.

3. Lind P, Igerc I, Beyer T, et al. Advantages and limitations of FDG PET in the follow-up of breast cancer. Eur J Nucl Med Mol Imaging 2004; 31: S125-134.

4. Chung A, Liou D, Karlan S, et al. Pre-operative FDG PET for axillary metastases in patients with breast cancer. Arch Surg 2006; 141: 783-789.

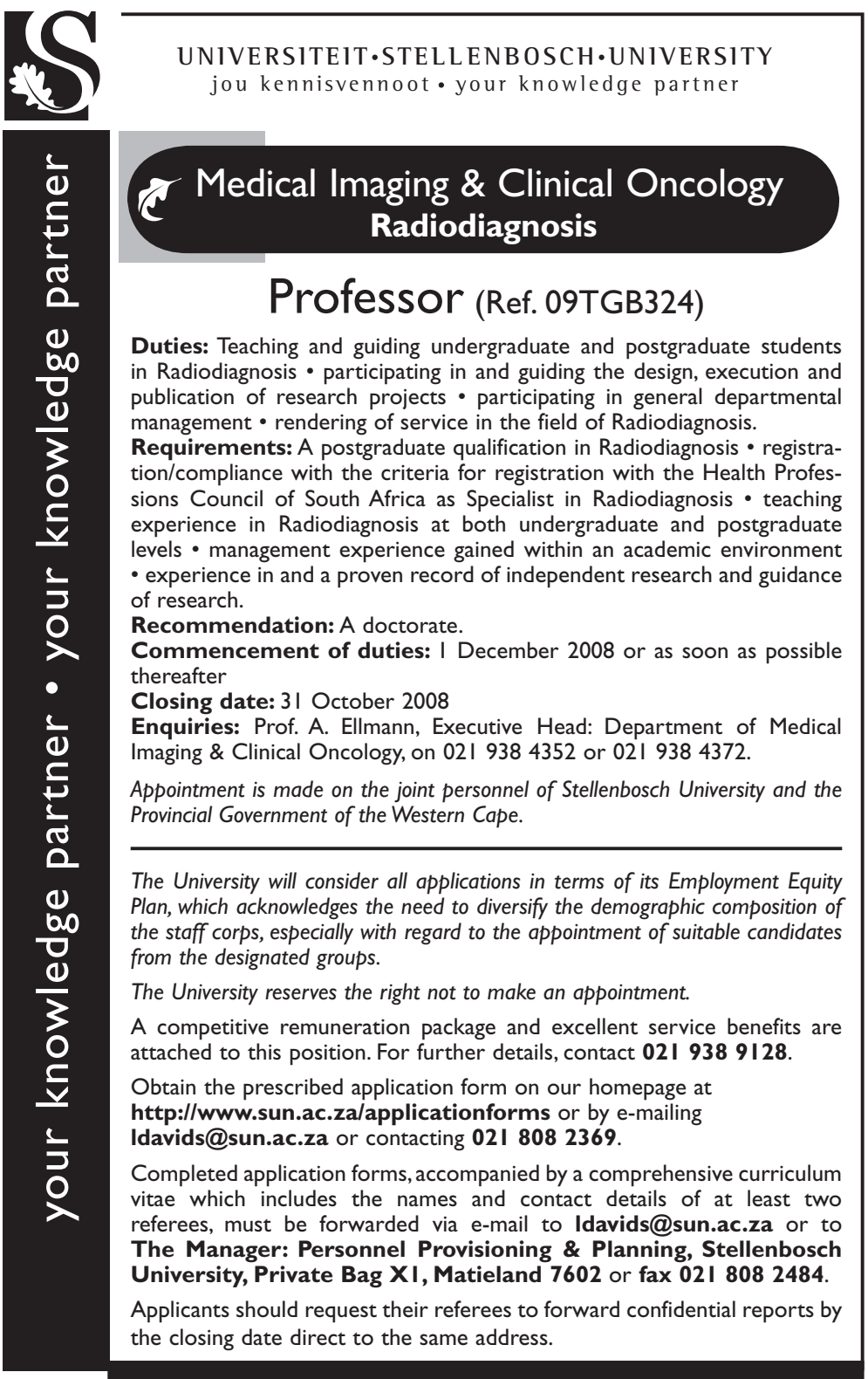

ayanda mbanga communications (cape) 15778 